NASA Technical Memorandum 106412

AIAA-93-4657

$$
|N-3|
$$

193078

$7 P$

\title{
Design and Implementation of a Status at a Glance User Interface for a Power Distribution Expert System
}

Eugene M. Liberman and David B. Manner

Sverdrup Technology, Inc.

Lewis Research Center Group

Brook Park, Ohio

and

James L. Dolce and Pamela A. Mellor

National Aeronautics and Space Administration

Lewis Research Center

Cleveland, Ohio

Prepared for the

AIAA: Computing in Aerospace 9

sponsored by American Institute of Aeronautics and Astronautics

San Diego, California, October 19-21, 1993

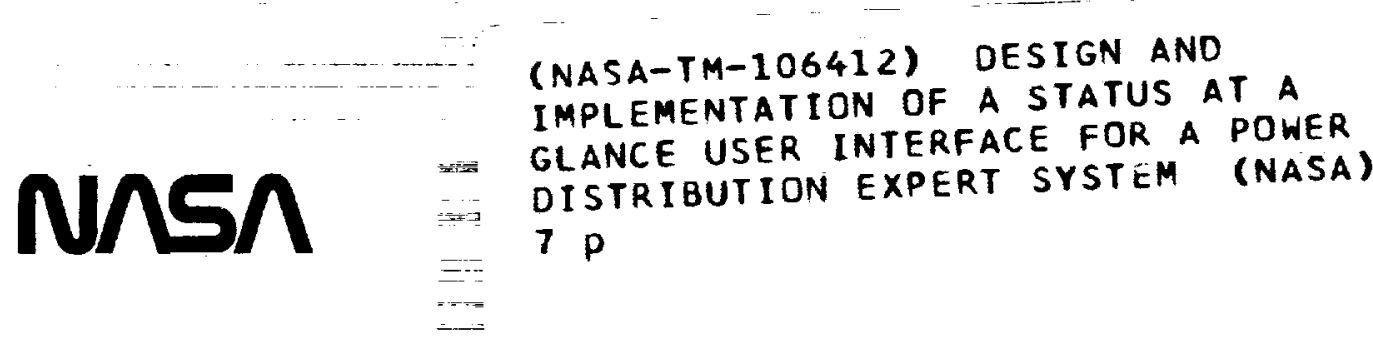





\section{DESIGN AND IMPLEMENTATION OF A STATUS AT A GLANCE USER INTERFACE FOR A POWER DISTRIBUTION EXPERT SYSTEM}

\author{
Eugene M. Liberman and David B. Manner \\ Sverdrup Technology, Inc. \\ Lewis Research Center Group \\ Brook Park, Ohio 44142
}

\begin{abstract}
Expert systems are widely used in health monitoring and fault detection applications. One of the key features of an expert system is that it possesses a large body of knowledge about the application for which it was designed. When the user consults this knowledge base, it is essential that the expert system's reasoning process and its conclusions be as concise as possible. If, in addition, an expert system is part of a process monitoring system, the expert system's conclusions must be combined with current events of the process. Under these circumstances, it is difficult for a user to absorb and respond to all the available information. For example, a user can become distracted and confused if two or more unrelated devices in different parts of the system require attention. A human interface designed to integrate expert system diagnoses with process data and to focus the user's attention to the important matters provides a solution to the "information overload" problem.
\end{abstract}

This paper will discuss a user interface to the power distribution expert system for Space Station Freedom. The importance of features which simplify assessing system status and which minimize navigating through layers of information will be discussed. Design rationale and implementation choices will also be presented.
James L. Dolce and Pamela A. Mellor National Aeronautics and Space Administration Lewis Research Center Cleveland, Ohio 44135

\subsection{INTRODUCTION}

The Space Station Freedom Power System is the most sophisticated power system aboard any spacecraft to date. Its behavior is monitored by on board systems as well as by specialists on the ground. When the bebavior is abnormal, diagnosing the cause can be tedious. Automating this task is necessary to ensure safe and efficient operation. Expert systems are excellent tools to provide this automation; however, an expert system alone cannot solve all the problems of monitoring and diagnostics. Human expertise is always required. The expert system needs to present information to the user without obfuscating the rest of the monitoring information. A well-designed user interface is the key to clearly both presenting system's status and expert system findings. The design of the user interface to the Space Station Freedom Power System expert system, Trouble, and the rationale for the design specifics will be discussed below.

\subsection{POWER SYSTEM OF THE SPACE STATION FREEDOM}

The Space Station Freedom requires electric power for its day-to-day operation while orbiting the earth. The power for the Station is obtained through converting solar energy into electricity, however, solar flux is not available throughout the station's orbit. To supply the station with power during the eclipse phase, excess power must be stored during the "sunny" portion of the orbit (insolation). Freedom's
Copyright c 1993 by the American Institute of Aeronautics and Astronautics, Inc. All rights reserved. 1 
power system consists of three subsystems: generation, storage, and distribution. The generation subsystem collects solar power during the insolation phase of an orbit using solar photovoltaic arrays to convert solar power into electric power. This generated electric power supplies energy to the consumer and is also stored in the storage subsystem for use during eclipse. The storage subsystem consists of rechargeable nickel-hydrogen batteries. The distribution subsystem carries power to many different types of power consumers ranging from a scientific experiment to an electric shaver used by an astronaut.

\subsection{POWER DISTRIBUTION SUBSYSTEM}

The power distribution subsystem is designed to supply power to the consumer. The power distribution subsystem components include Remote Bus Isolators (RBI), Remote Power Controllers (RPC), and various buses and cables connecting RBIs and RPCs. These components and their interconnections form the power distribution network. The distribution network and its hardware is protected by circuit breakers - the RBI's and the RPC's - which cut off power to overloads. If current flowing through a circuit breaker exceeds its maximum allowable value, the circuit breaker trips interrupting the flow of power down the line.

The inherent complexity of this system can lead to problems. Operating with insufficient margin between the line current and the breaker settings may cause inadvertent power loss or the protection hardware itself may fail. A human alone cannot be expected to deal with a system this complex. An expert system assistant can provide explanations of encountered faults and operating advice. Such an expert system, combined with a well-designed user interface, simplifies the user's task of understanding the status of the power distribution subsystem. The user is able to see "at a glance" what the current status is and quickly pinpoint the problem area, leading to faster and more accurate problem resolution.

\subsection{THE POWER DISTRIBUTION EXPER} SYSTEM

The power distribution expert system, Trouble, was developed at NASA Lewis Research Center in Cleveland, $\mathrm{OH}$ to monitor and diagnose faults in the power distribution subsystem described above. Trouble is an object-oriented, rule-based, set-covering expert system written in ART (Automated Reasoning Tool), an expert system shell by Inference corporation and LISP running on a Texas Instrument Explorer II workstation. Trouble is also a multiprocess system that consists of the following independent processes: data acquisition, symptom detection, diagnosis, and graphical user interface. Data acquisition is responsible for obtaining the latest values from the power distribution subsystem and updating the corresponding objects in Trouble. Symptom detection is a set of rules that determine whether or not an anomaly is present in the system. Diagnosis is responsible for taking the generated symptoms and searching the failure database to explain an anomaly's cause. The graphical user interface communicates with the user. ${ }^{1}$

\subsection{USER INTERFACE}

\subsection{Implementation details}

The user interface is built using the Transportable Application Environment (TAE +) software package developed by NASA Goddard Space Flight Center (GSFC). The user interface runs on a SUN 
SparcStation 2 employing Motif under Unix with the Ada language used for programming. The expert system resides on a completely different computer, so a method for sending information between different machines was needed. A socket-to-socket communication protocol and Remote Procedure Call (RPC) protocol were the top contenders. Both protocols were implemented, but RPC was the final choice due to its reliability and conservative use of CPU cycles. TAE + was selected for its quick prototyping and Ada programming language capabilities.

The user interface screens are designed with TAE+'s drawing package. Once the screens are drafted they are exercised using TAE+'s rehearsal mode. This mode animates the dynamic portions of the screens so that each screen's effectiveness can be judged and refined early in the development process. After the screens are completed to our satisfaction, TAE + generates source code that reflects the designed screens. (TAE + offers a choice of several languages.) The source code generated only animates the icons. The next step is to add code to acquire and to display data as well as to respond to the user's inputs.

The TAE + environment is well-equipped to handle user input; however, data coming from the expert system had to be handled separately. The RPC protocol is used to receive data from the expert system. The expert system determines what remote procedure to call based on the data that needs to be transmitted. The data is sent as an input parameter to a remote procedure. Remote procedures on the user interface side evaluate the data sent and determine what action to take based on the type of data received, e.g. update alpha-numeric values or change colors.

\subsection{Screen layout}

The user interface for the power distribution expert system is designed from the schematic diagram of the power distribution subsystem. The diagram begins at the top with the Roll Rings, a rotary joint connecting the power distribution subsystem with the power generation and storage subsystems. The devices depicted on the schematic include circuit breakers (RBIs and RPCs), buses, loads, and lines connecting the devices.

The power schematic bas been oriented such that power is shown to flow downhill, e.g. from the top of the screen with the roll rings to the loads at the bottom. These power components form a power distribution tree. The top-down perspective conveys to users the connectivity as well as the direction of power flow. As a result, terms such as up-stream and down-stream are easier to understand. For example, if an up-stream circuit breaker is open, (i.e. the immediate parent) then all breakers below the parent (descendants) will not be energized. This notion of top-down power hierarchy was chosen because it capitalizes on everyone's intuitive understanding of gravity.

Another portion of the screen is reserved for control buttons. These buttons control the amount of information displayed upon the screen. Some information may not be required at all times, especially in the normal operating mode. The buttons provide the means for hiding or showing information. The button names serve as legends for the button's control function. Reducing clutter on the screen creates a more comfortable monitoring environment. The buttons are color coded to match the colors of the screen information that they control. For example, the gold button controls the display of the 
names of the devices, therefore, the devices' names are displayed in gold. This feature makes the association between the button and its data readily apparent.

Another portion of the screen is dedicated to text messages. It is called the annunciator and its purpose is to explain abnormalities if they occur. The annunciator is always present and cannot be hidden. The messages that appear on the annunciator are one line descriptions for each anomaly detected by the expert system. When an operator selects a particular line in the annunciator, that line is highlighted to emphasize the selection. At the same time, a purple arrow appears next to the device on the schematic diagram that bas detected the anomaly. Selection of an anomaly line also opens up a text area that contains a more detailed description of the problem. This detailed description appears in its own portion of the screen above the annunciator. This text is visible only when the annunciator line is selected, and it does not obscure the schematic ${ }^{2}$. The highlighted line on the annunciator, the purple arrow on the schematic diagram, and the detailed explanation of the abnormality link the message, the device, and the explanation together. These visual cues make it easy for the user to see the relationship among the messages and the icons. When a message appears on the annunciator, an acknowledge button is also activated. This makes the user aware that there is a new message present, a fact that might be obscured in an all text area of the screen.

\subsection{Graphical description of components}

The Space Station Freedom Program (SSFP) Flight Human-Computer Interface Standard describes certain standards to be applied to all the SSFP-related userinterfaces ${ }^{3}$. The requirements were adopted. The icons describing lines or cables were depicted as straight lines and the buses as thicker straight lines. The shapes and the size of circuit breakers and load icons were also specified. The sizes specified for the circuit breaker and load icons, however, were too large for the complexity of our distribution schematic. We preserved the design of the icons and reduced the sizes from the standards. To assist the user in the navigation of the interface, the graphical elements were divided into two groups, "clickable" and "non-clickable". Clickable elements change the user interface when the element is selected by clicking on it with a mouse button. The clickable components are the buttons, the obvious choice for clicking, and the lists of text -- not so obvious. The buttons on the screen are all the same size and shape to avoid confusion. The clickable lists of text, the annunciator, and the detailed display window have messages emphasizing the clickability of these items. The rest of the graphical elements on the screen are not clickable.

\subsection{Volume of information and color and size} relation.

The less cluttered the user interface screens, the more effective the screen design. One way to reduce clutter is to convey some information by colors and sizes as opposed to messages and numbers. The power distribution system delivers power to the required loads through a hierarchy of the cables (lines), circuit breakers, and busses. Colors are used to convey the notion of power flow and electrical potential. A dim green color shows the components that are not energized while a bright green color shows those that are energized. The user can clearly see the path that the power takes to get to the scheduled load. Tripping a circuit breaker causes all the devices down-stream to lose power causing those components to change 
from bright to dim green. An energized component has voltage but not necessarily current. When a load is connected and operating, current flows through the distribution network. Current is depicted using a bar graph that resembles a thermometer. The graph fills up, from bottom to top, as the current increases. The top of the graph represents the breaker's setpoint for tripping on over-current. Thus the graph shows the operating margin which can be determined with a quick glance.

\subsection{Size of alphanumeric characters}

Numeric values are provided for currents, breaker trip values, and voltages. The characters are small compared with the power component icons because they are of secondary importance to the operator. The current meters and the highlighted path of energized components dominate the display and provide the essential monitoring information under normal operating conditions. The screen can be viewed from a distance or quickly scanned at a glance without the need to know the actual numbers representing the system's status. When an anomaly occurs, the user naturally concentrates on the display, taking a closer look at the numerical values. Thus numeric information does not clutter the screen but is available when greater detail is needed.

\subsection{Salience using color and size}

The "status-at-a-glance" user interface design strives to control the focus of the user's attention. Color salience is one way to make an object distinguishable from the rest. Color is used to emphasize the energized components: the bright florescent green components clearly stand out from the dim green ones. Similarly, the annunciator window is bright grey which is clearly distinguishable from the dark background color. Another way of drawing the user's attention to a graphical object is with the size. Size salience is used to focus attention on components exhibiting anomalous behavior. An anomaly detected in a circuit breaker, for example, is depicted by drawing a larger box around the faulty device.

\section{$\underline{6.0 \text { CONCLUSION }}$}

The amalgamation of these design features, namely: message linking arrows, reduced information content screens, high salience anomaly icons, and color choices with failure detection and diagnostic explanation from an expert system provides an effective status-at-a-glance monitoring system for power distribution. This user interface design presents diagnostic reasoning without compromising the monitoring of current events. The display can convey complex concepts in terms that are clear to its users.

\section{REFERENCES}

1. D.B. Manner, E.M. Liberman, P.A. Mellor and J.L. Dolce, "Reducing the Cognitive Workload: Trouble Managing Power Systems," in Proc. 9th Computing in Aerospace Conference, AIAA 1, (1993).

2. D.D. Woods, "The Cognitive Engineering of Problem Representation," Human-Computer Interaction and Complex Systems, London, Academic Press, (1991).

3. Space Station Freedom Program Flight HumanComputer Interface Standards, SSP 30570, draft September 1991 


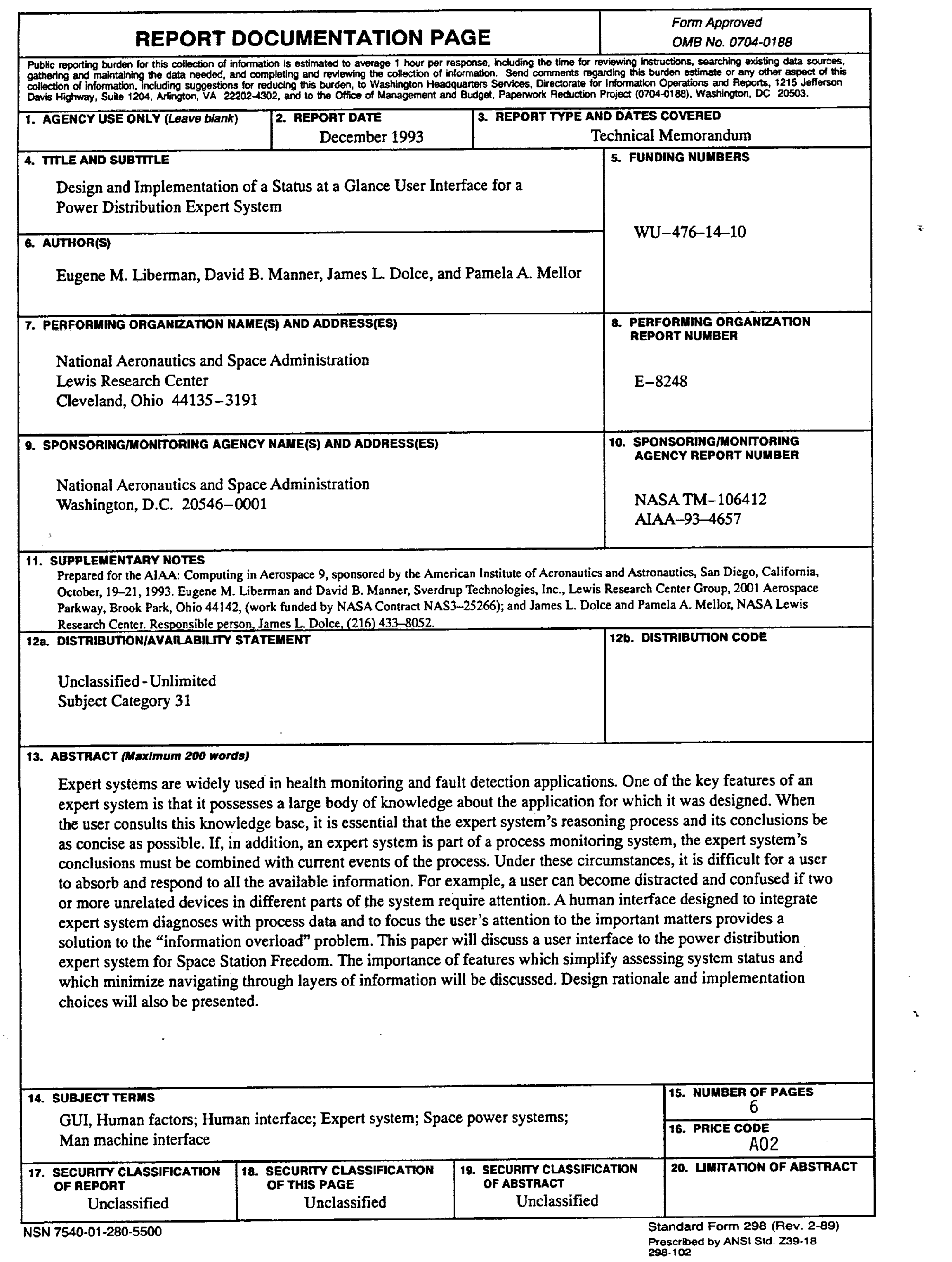

